\title{
一锅两步法合成 1,4-二芳基取代-1,3-丁二烯类化合物
}

\author{
梁静茹 王冰荣 ${ }^{\dagger}$ 黄成尹 叶晓君温燕梅* \\ (广东海洋大学化学与环境学院 广东湛江 524088)
}

\begin{abstract}
摘要 1,3-共轭烯烃不仅是有机合成不可或缺的原料或中间体，许多聚合物、天然产物和具有生理活性化合物的核心骨 架都含有 1,3-丁二烯结构单元. 简便而有效的 1,3-共轭烯烃合成方法一直是化学家们感兴趣的研究课题, 在已有共轭烯 烃的合成方法中，钯催化烯基卤与端烯的 Heck 交叉偶联反应占据了主要地位. 最近，简单化合物的 “一锅法” 串联反 应构建碳碳键具有操作及反应后处理简单、原子经济性高等优点而备受关注. 以价廉易得端炔和联硼酸频哪醇酯为原 料, 在碱催化下进行嗍氢化反应生成烯基硼酸频哪醇酯, 这一中间体无需分离纯化，在 $\operatorname{Pd}(\mathrm{OAc})_{2}$ 和碱的作用下实现一 锅串联反应得到目标产物 1,4-二芳基取代-1,3-丁二烯化合物, 所有化合物的结构用 ${ }^{1} \mathrm{H} N \mathrm{NMR}$ 和 ${ }^{13} \mathrm{C} \mathrm{NMR}$ 进行表征, 该 一锅串联法合成途径简捷, 反应条件温和，为共轭烯烃化合物合成提供了简便的途径.
\end{abstract}

关键词 一锅两步法; 端炔; 烯基硼酸酯; 1,3-丁二烯

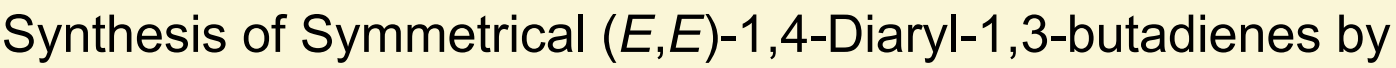 One-Pot Method}

\author{
Liang, Jingru ${ }^{\dagger} \quad$ Wang, Bingying $^{\dagger} \quad$ Huang, Chengyin $\quad$ Ye, Xiaojun $\quad$ Wen, Yanmei* \\ (Faculty of Chemistry and Environmental Science, Guangdong Ocean University, Zhanjiang, Guangdong 524088)
}

\begin{abstract}
Conjugated olefins are very useful synthetic intermediates. Furthermore, 1,3-diene moiety is an important unit often found in many polymers, natural products and biologically active compounds. For these reasons, the design and synthesis of compounds containing a diene moiety have received considerable attention in organic synthesis and have been extensively studied. Among these methods, the Heck cross-coupling reaction between alkenyl halide and terminal alkene under palladium catalysis has become a powerful tool for the synthesis of dienes. Recently, many research groups have focused on "one-pot method" cascade reactions to form carbon-carbon bond due to not only their convenient and simple operation, but also their high atomic economy. Using terminal alkyne and bis(pinacolato)diboron as starting materials and $\mathrm{NaOMe}$ as the base, alkenyl borates were formed, and a series of 1,3-butadienes were directly synthesized via palladium-catalyzed homocoupling reaction of alkenyl borates. The structures of all the compounds were confirmed by ${ }^{1} \mathrm{H}$ NMR and ${ }^{13} \mathrm{C}$ NMR. This one pot method is simple and mild, which provides a simple way for the synthesis of a series of 1,3-butadienes.
\end{abstract}

Keywords one-pot method; terminal alkyne; alkenyl borate; 1,3-butadiene

1,3-丁二烯类化合物结构中含有相互共轭的 $\pi-\pi$ 双 键，是一种十分独特的有机分子，含有 1,3-丁二烯结构 单元的化合物是非常重要的有机合成砌块, 很多具有生 物活性的天然产物和药物都包含了 1,3-丁二烯结构单 元, 1,3-丁二烯衍生物还是合成许多重要药物的关键中 间体. 共轭二烯烃中 1,4-二芳基-1,3-丁二烯类化合物由
于具有特殊的光学性质, 在液晶和发光体等化学材料中 有着较为广泛的应用. 因此, 如何有效合成含有 1,3-丁 二烯结构的化合物是近年来化学家们的研究热点, $1,3-$ 丁二烯类化合物的合成方法以及应用一直受到人们的 关注 ${ }^{[1]}$.

有关 1,3-丁二烯衍生物的合成有许多文献报道, 1,3-

\footnotetext{
* Corresponding author. E-mail: wym1503@163.com

Received October 7, 2020; revised December 16, 2020; published online January 14, 2021.

Project supported by the Project of Enhancing School with Innovation of Guangdong Ocean University (No. 230419054), the College Students Innovation Program of Guangdong Ocean University (No. 580520106) and the Graduate Education Innovation Program of Guangdong Ocean University (No. 521002082).

广东海洋大学创新强校工程(No. 230419054)、广东海洋大学大学生创新训练(No. 580520106)、广东海洋大学学位与研究生教育改革研究(No. 521002082)资助项目.

†共同第一作者(These authors contributed equally to this work).
} 
丁二烯类化合物的合成方法大致可以分为两类, 一类是 传统的交叉偶联反应 ${ }^{[2]}$, 如 $\alpha, \beta$-不饱和羰基化合物的烯 基化 Wittig 反应(Scheme 1,a)、烯基卤的 Heck 反应、烯 基卤与有机锌试剂 Negishi 反应、烯基卤与有机硼试剂 Suzuki 反应等(Scheme 1, b), 传统的交叉偶联反应需要 制备官能团化的反应底物的额外反应步骤, 不是预先合 成卤代烯烃就是预先合成金属有机试剂, 存在合成方法 复杂、反应路线长和反应副产物多等问题. 另一类反应 是过渡金属催化碳氢键作为反应的官能团直接形成 C$\mathrm{C}$ 键, 主要包括官能团导向的烯烃与末端烯烃的氧化交 叉偶联(Scheme 1, c ) ${ }^{[3]}$, 还有几例未活化端烯直接自身 偶联 ${ }^{[4]}$, 但该反应的适用范围十分有限. 此外, 还包括 以硅为基础的烯化反应 ${ }^{[5]}$ 、炔烃与烯烃的亲核钯化/Heck 串联反应 ${ }^{[6]}$ 以及卤代烃、芳炔与末端烯三组分串联反应 等 ${ }^{[7]}$. 无论是传统的交叉偶联反应还是碳氢活化的氧化 脱氢偶联都难以合成 1,4-二芳杂环-1,3-丁二烯类化合 物, 有关合成噻吩、吡啶类取代-1,3-丁二烯的文献报道 极少, Itami 小组 ${ }^{[8]}$ 巧妙地设计了乙烯基(2-吡啶)硅烷和 芳卤发生 Mizoroki-Heck 反应, 然后生成的中间体再发 生自身偶联反应 1,4-二噻吩基-1,3-丁二烯.

(a) Wittig reaction using $\alpha, \beta$-unsaturated aldehydes:

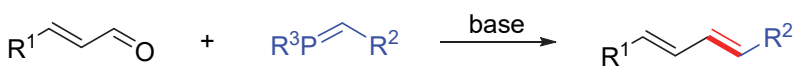

(b) Cross-couplings of alkenyl organometallics and alkenyl halides:

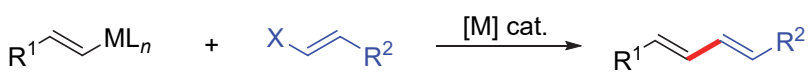

$\mathrm{M}=\mathrm{B}, \mathrm{Zn}, \mathrm{Al}$, etc.; $\mathrm{L}=$ ligand, $\mathrm{X}=\mathrm{I}, \mathrm{Br}$, etc.

(c) Directing group enabled oxidative couplings between two olefins:

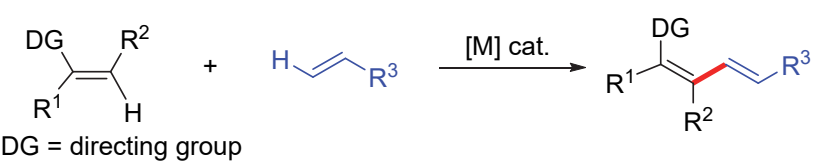

图式 1 合成 1,3-丁二烯的典型方法

Scheme 1 Typical approaches for the synthesis of 1,3-butadiene

随着绿色化学的发展, 合成 1,4-二芳基-1,3-丁二烯 的传统方法显现出越来越多的局限性, 如重金属离子污 染、反应步骤多及产率低等, 因此, 寻找更为高效和环 境友好的合成路径是十分必要的. 烯基硼酸酯是一类重 要的有机合成中间体, 此类化合物不仅广泛用于和芳 基、烷基亲电试剂的 Suzuki-Miyaura交叉偶联反应 ${ }^{[9]}$, 而 且还常常作为合成二芳基化合物的偶联试剂 [10]. 烯基 嗍酸酯比其他的碳有机金属试剂具有更好的稳定性, 能 通过简单的色谱分离进行纯化并咜存于空气中. 因此, 采用各种不同的方法合成烯基硼化合物一直是化学家 们感兴趣的研究课题, 最近, 无金属无配体条件下的嗍
化反应更是受到人们的关注 ${ }^{[11]}$. 我们以价廉易得端炔、 联硼酸频哪醇酯为原料, 在碱催化下进行硼氢化反应可 以生成烯基嗍酸频哪醇酯 ${ }^{[2]}$. 在此基础上，以中间体烯 基硼酸频哪醇酯为原料，在 $\mathrm{Pd}(\mathrm{OAc})_{2}$ 和碱的条件下发 生自身偶联得到目标产物 1,4-二芳基-1,3-丁二烯化合 物, 实现了以端炔为原料, 通过一锅两步串联法构建 $\mathrm{C}\left(\mathrm{sp}^{2}\right)-\mathrm{C}\left(\mathrm{sp}^{2}\right)$ 键，该方法简捷，反应条件温和，为合成 共轭烯烃化合物提供了简便的途径.

\section{1 结果与讨论}

\section{1 炔烃嗍氢化生成烯基硼酸频哪醇酯}

有机硼化物是一类重要的有机合成中间体. 近年 来，有机硼试剂不仅广泛用于与芳基、烷基亲电试剂的 Suzuki-Miyaura 交叉偶联反应，而且还常常作为氧化交 叉偶联反应的烃基化试剂, 因此, 合成具有各种结构的 有机硼化合物一直是化学家们感兴趣的研究课题. 在研 究不饱和烃与联硼酸频哪醇酯反应时, 我们发现, 以 $\mathrm{NaOMe}$ 为碱，在甲醇溶剂中, $40{ }^{\circ} \mathrm{C}$ 搅拌反应 $10 \mathrm{~h}$ 可以 实现炔烃的嗍氢化反应生成烯基硼酸频哪醇酯(表 1$)^{[12]}$.

表 1 炔烃与联硼酸频哪醇酯反应的结果

Table 1 Hydroboration of different alkynes with bis(pinacolato)diboron
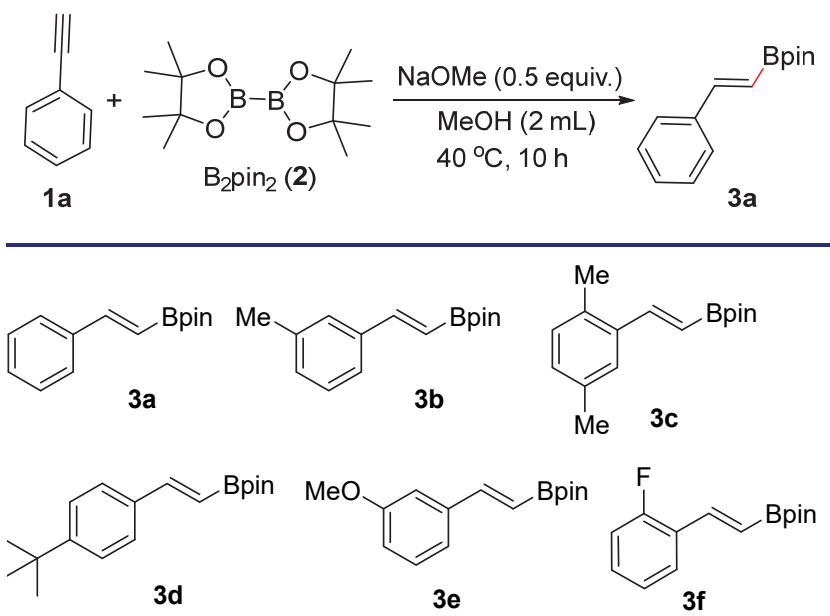

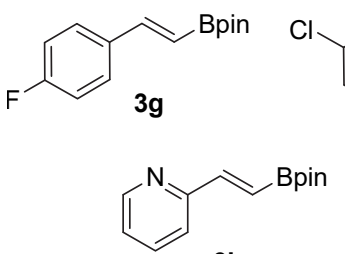

$3 \mathbf{j}$

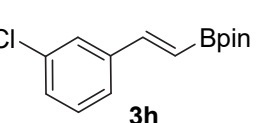

$3 h$<smiles>Brc1ccccc1</smiles>

3k
1.2 烯基硼酸频哪醇酯自身偶联生成 1,3-丁二烯化合 物

为了证实炔烃硼氢化生成烯基硼酸频哪醇酯的有 用性，探索了它的潜在应用前景. 以 $\mathrm{Pd}(\mathrm{OAc})_{2}$ 为催化 
剂, $\mathrm{Na}_{2} \mathrm{CO}_{3}$ 为碱, 于 $\mathrm{N}, \mathrm{N}$-二甲基乙酰胺溶剂中 $40{ }^{\circ} \mathrm{C}$ 反 应 $6 \mathrm{~h}$, 烯基硼酸频哪醇酯可以进行分子氧参与的钯催 化自身偶联反应得到 1,4-二苯基-1,3-丁二烯(Eq. 1).

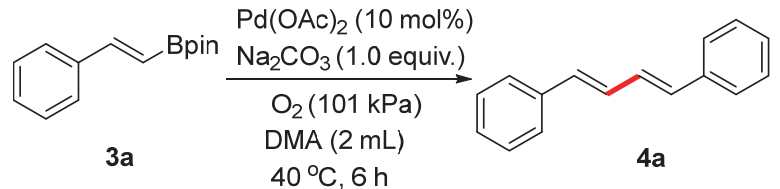

\section{3 炔烃硼氢化/自身偶联一锅法串联合成 1,4-二芳基} 取代-1,3-丁二烯化合物

采用端炔为前体的 “一锅法” 反应，首先将联硼酸 频哪醇酯与苯乙炔在甲醇中 $40{ }^{\circ} \mathrm{C}$ 反应 $10 \mathrm{~h}$ 得到烯基硼 酸频哪醇酯，然后，向反应体系中加入 $\mathrm{Pd}(\mathrm{OAc})_{2}$ 、 $\mathrm{Na}_{2} \mathrm{CO}_{3} 、 N, N$-二甲基乙酰胺(DMA)，于 $40{ }^{\circ} \mathrm{C}$ 反应 $6 \mathrm{~h}$ 得到目标产物. 通过实验发现, 直接以端炔为原料也可 以一锅合成对称的 1,4-二芳杂环-1,3-丁二烯类化合物. 如表 2 所示, 一系列取代的末端炔都能以中等到较好的 收率获得目标产物共轭二烯. 进一步底物探索发现, 底 物取代基位置对该氧化偶联反应没有特别的影响, 邻位 (1f)、间位(1b,1e)、对位(1d)烷基和卤素 $(1 \mathrm{~g}, 1 \mathrm{~h})$ 的末端 炔均能较好地进行反应, 甚至多取代芳环末端炔(1c)也 能一锅法合成共轭二烯产物. 考虑到共轭二烯在功能材 料领域的重要应用潜能, 以及本反应兼容的芳基卤(1h) 可以很好地进行金属催化的交叉偶联反应, 所得目标产 物可以进一步转化为功能材料的关键骨架. 更为重要的 是，传统的基于金属催化偶联反应来构建共轭二烯的反 应中, 吡啶杂环活性较差, 甚至在催化体系中难以兼容. 本转化中基于钯催化氧化偶联的一锅法反应构建共轭

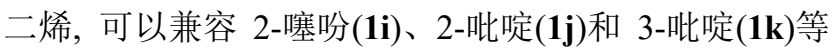
杂环取代基, 从而为这类常规方法难以获得的产物提供 简捷的合成方法.

有趣的是, 对于强供电子基团甲氧基取代的烯基硼 酸酯 31 底物, 其在二价钯催化, 化学计量的醋酸铜为氧 化剂条件下, 得到的是支链状的共轭二烯产物 51 (Eq. 2), 而非使用氧气作为最终的氧化剂对应的直链状的共 轭二烯. 需要指出的是, 通过氧化偶联反应构建这类支 链状共轭二烯很有研究价值 ${ }^{[13]}$, 进一步的机理探讨和 应用拓展正在进行中。<smiles>COc1ccc(/C=C/B2OC(C)(C)C(C)(C)O2)cc1</smiles>

$$
\begin{gathered}
\underset{\mathrm{Pd}(\mathrm{OAc})_{2}(10 \text { mol\%) }}{\left.\mathrm{Na}_{2} \mathrm{CO}_{3} \text { (1.0 equiv. }\right)} \\
\underset{\mathrm{Cu}(\mathrm{OAc})_{2}(2.0 \text { equiv. })}{\mathrm{O}_{2}(101 \mathrm{kPa})}
\end{gathered}
$$

DMA $(2 \mathrm{~mL}), 40^{\circ} \mathrm{C}, 6 \mathrm{~h}$<smiles>C=C(/C=C/c1ccc(OC)cc1)c1ccc(OC)cc1</smiles>

表 2 炔烃硼氢化/自身偶联一锅法串联反应的结果 ${ }^{a}$

Table 2 The cascade reactions of hydroboration of alkynes and homocoupling of alkenyl borates
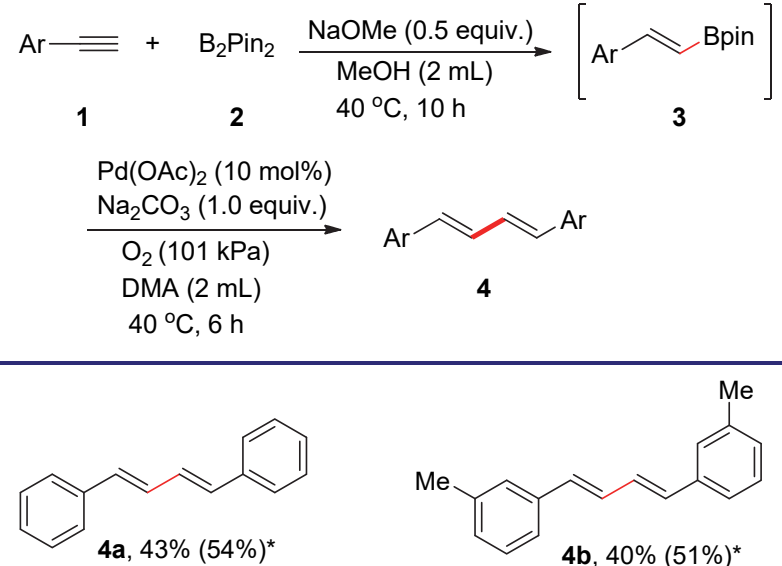<smiles>Cc1cccc(/C=C/C=C/c2cccc(C)c2)c1</smiles><smiles>Cc1ccc(C)c(/C=C/C=C/c2cc(C)ccc2C)c1</smiles>

Me $4 c, 47 \%(59 \%)^{*}$ tBu<smiles>CC(C)(C)c1ccc(/C=C/C=C/c2ccc(C(C)(C)C)cc2)cc1</smiles>

4d, $56 \%(71 \%)^{*}$<smiles>COc1cccc(/C=C/C=C/c2cccc(OC)c2)c1</smiles>

$4 e, 63 \%(79 \%)$<smiles>Fc1ccc(/C=C/C=C/c2ccc(F)cc2)cc1</smiles>

$4 \mathrm{~g}, 68 \%(80 \%)^{*}$<smiles>C(/C=C/c1cccs1)=C\c1cccs1</smiles>

$4 \mathbf{i}, 35 \%(44 \%)^{*}$<smiles>CC(C)(C)c1cccnc1</smiles>

$\mathbf{4 k}, 33 \%(41 \%)^{*}$ 4f, $64 \%(75 \%)^{*}$<smiles>Clc1cccc(/C=C/C=C/c2cccc(Cl)c2)c1</smiles>

4h, $61 \%(72 \%)^{\star}$

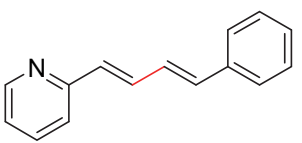

$4 j, 30 \%(38 \%)^{\star}$
${ }^{a}$ Isolated yields, the corresponding yield of the target product obtained from alkenyl borate in brackets.

\section{2 结论}

以端炔和联硼酸频哪醇酯为原料, 在碱催化下进行 喼氢化反应生成烯基硼酸频哪醇酯，该中间产物不需要 分离, 加入醋酸钯为催化剂, 碳酸钠为助剂, 经自身偶 联实现一锅两步反应高选择性生成 1,4-二芳基取代-1,3丁二烯产物。该一锅法串联反应具有原料易得、操作简 便及中间产物不需要分离等优点，更重要的是以价廉易 得的原料可以合成 1,4-二芳杂环-1,3-丁二烯类化合物. 


\section{3 实验部分}

\section{1 仪器与试剂}

德国 Bruker 公司 $400 \mathrm{MHz}$ DRX-400 核磁共振仪, TMS 为内标; 美国 Frinnigan 公司 Trace DSQ GC-MS 气 质联用仪[色谱柱: 高弹性石英毛细柱 HP-5, $0.2 \mathrm{~mm} \times$ $0.25 \mu \mathrm{m} \times 30 \mathrm{~mm}$, EI 源(电子能量: $70 \mathrm{eV}$ )]. 所用的化学 原料试剂全部从 Alfa, Aldrich 等公司购买, 没有进一步 的纯化; 常用溶剂购自广州试剂公司; 薄层层析硅胶由 硅胶 GF254(青岛海洋化工厂)和羧甲基纤维素钠自制自 制; 柱层析用硅胶为化学纯试剂(青岛海洋化工厂).

\section{2 实验方法}

往试管中相继加入 $\mathrm{NaOMe}(0.5$ equiv.)、联硼酸频哪 醇酯 $(1 \mathrm{mmol})$ 、端㶧 $(1 \mathrm{mmol})$ 和甲醇 $(2 \sim 3 \mathrm{~mL})$, 将装有 反应混合液的试管置于 $40{ }^{\circ} \mathrm{C}$ 油浴中搅拌反应 $10 \mathrm{~h}$. 用 TLC 检测反应完成后, 冷却至室温, 往反应混合液中加 入 $9 \sim 10 \mathrm{~mL}$ 饱和食盐水溶液和 $6 \sim 8 \mathrm{~mL}$ 乙酸乙酯, 连 续用乙酸乙酯萃取三次, 收集有机相, 有机相用无水 $\mathrm{MgSO}_{4}$ 干燥, 抽滤、减压蒸馏除去溶剂, 产物经 GC-MS 进行分子量确认. 接着在上述反应生成的烯基硼酸频哪 醇酯中加入 $\mathrm{Pd}(\mathrm{OAc})_{2}(10 \mathrm{~mol} \%) 、 \mathrm{Na}_{2} \mathrm{CO}_{3}$ (1 equiv.) 和 DMA $(2 \mathrm{~mL})$, 接好充有氧气的气球, 将装有反应混合 液的试管置于 $40{ }^{\circ} \mathrm{C}$ 油浴中搅拌反应 $6 \mathrm{~h}$. 用 TLC 检测反 应完成后, 冷却至室温. 经制备薄层色谱分离、抽滤、 减压蒸馏后可得目标产物. 产物的结构经 GC-MS、 ${ }^{1} \mathrm{H}$ NMR 和 ${ }^{13} \mathrm{C}$ NMR 进行分析确认.

反, 反-1,4-二苯基-1,3-丁二烯 $(\mathbf{4 a})^{[14]}$ : 白色固体, 收 率 43\%, m.p. $143 \sim 145{ }^{\circ} \mathrm{C}$ (lit. ${ }^{[14]} 143{ }^{\circ} \mathrm{C}$ ); ${ }^{1} \mathrm{H}$ NMR (500 $\left.\mathrm{MHz}, \mathrm{CDCl}_{3}\right) \delta: 7.45(\mathrm{~d}, J=7.5 \mathrm{~Hz}, 4 \mathrm{H}), 7.34$ (t, $J=7.5$ $\mathrm{Hz}, 4 \mathrm{H}), 7.26 \sim 7.23(\mathrm{~m}, 2 \mathrm{H}), 7.00 \sim 6.94(\mathrm{~m}, 2 \mathrm{H}), 6.72 \sim$ $6.66(\mathrm{~m}, 2 \mathrm{H}) ;{ }^{13} \mathrm{C}$ NMR $\left(126 \mathrm{MHz}, \mathrm{CDCl}_{3}\right) \delta: 137.3$, $132.8,129.3,128.7,127.6,126.4$.

反, 反-1,4-二间甲苯基-1,3-丁二烯(4b) ${ }^{[15]}$ : 白色固 体, 收率 40\%, m.p. $163 \sim 167{ }^{\circ} \mathrm{C}$ (lit. ${ }^{[15]} 78{ }^{\circ} \mathrm{C}$ ); ${ }^{1} \mathrm{H}$ NMR $\left(500 \mathrm{MHz}, \mathrm{CDCl}_{3}\right) \delta: 7.29 \sim 7.28(\mathrm{~m}, 3 \mathrm{H}), 7.26 \sim 7.23(\mathrm{~m}$, $3 \mathrm{H}), 7.08(\mathrm{~d}, J=7.0 \mathrm{~Hz}, 2 \mathrm{H}), 7.00 \sim 6.94(\mathrm{~m}, 2 \mathrm{H}), 6.69 \sim$ $6.63(\mathrm{~m}, 2 \mathrm{H}), 2.39(\mathrm{~s}, 6 \mathrm{H}) ;{ }^{13} \mathrm{C}$ NMR $\left(126 \mathrm{MHz}, \mathrm{CDCl}_{3}\right) \delta$ : $138.2,137.3,132.8,129.2,128.6,128.4,127.1,123.6$, 21.5 .

反, 反-1,4-二(2,5-二甲基苯基)-1,3-丁二烯(4c) ${ }^{[16]}$ : 白 色固体, 收率 47\%, m.p. 108 120 ${ }^{\circ} \mathrm{C}$ (lit. ${ }^{[16]} 150 \sim$ $\left.152{ }^{\circ} \mathrm{C}\right) ;{ }^{1} \mathrm{H}$ NMR $\left(500 \mathrm{MHz}, \mathrm{CDCl}_{3}\right) \delta: 7.40(\mathrm{~s}, 2 \mathrm{H}), 7.07$ (s, 2H), $7.05 \sim 6.86(\mathrm{~m}, 6 \mathrm{H}) ;{ }^{13} \mathrm{C} \mathrm{NMR}\left(126 \mathrm{MHz}, \mathrm{CDCl}_{3}\right)$ $\delta: 136.0,135.4,132.6,130.5,130.4,130.2,128.3,125.6$, 21.1, 19.4.
反, 反-1,4-二(4-叔丁基苯基)-1,3-丁二烯(4d $)^{[17]}$ : 白 色固体, 收率 $56 \%$, m.p. $121 \sim 123{ }^{\circ} \mathrm{C}$ (lit. ${ }^{[17]} 173 \sim$ $\left.176{ }^{\circ} \mathrm{C}\right) ;{ }^{1} \mathrm{H}$ NMR $\left(500 \mathrm{MHz}, \mathrm{CDCl}_{3}\right) \delta: 7.40 \sim 7.35(\mathrm{~m}$, $8 \mathrm{H}), 6.96 \sim 6.90(\mathrm{~m}, 2 \mathrm{H}), 6.68 \sim 6.62(\mathrm{~m}, 2 \mathrm{H}) ;{ }^{13} \mathrm{C} \mathrm{NMR}$ $\left(126 \mathrm{MHz}, \mathrm{CDCl}_{3}\right) \delta: 150.6,134.7,132.2,128.8,126.1$, 125.6, 34.6, 31.3.

反, 反-1,4-二(3-甲氧基苯基)-1,3-丁二烯(4e $)^{[17] \text { : 白 }}$ 色固体, 收率 $63 \%$, m.p. $153 \sim 155{ }^{\circ} \mathrm{C}\left(\right.$ lit. ${ }^{[17]}$ no report); ${ }^{1} \mathrm{H}$ NMR $\left(400 \mathrm{MHz}, \mathrm{CDCl}_{3}\right) \delta: 7.28(\mathrm{~d}, J=7.6 \mathrm{~Hz}, 4 \mathrm{H})$, 7.09 (d, $J=14.0 \mathrm{~Hz}, 4 \mathrm{H}), 6.90$ (d, $J=7.2 \mathrm{~Hz}, 4 \mathrm{H}), 6.82$ (d, $J=8.8 \mathrm{~Hz}, 1 \mathrm{H}), 6.65(\mathrm{dd}, J=1.6,14.0 \mathrm{~Hz}, 1 \mathrm{H}), 6.54(\mathrm{~d}$, $J=11.6 \mathrm{~Hz}, 1 \mathrm{H}), 3.86(\mathrm{~s}, 6 \mathrm{H}) ;{ }^{13} \mathrm{C}$ NMR $(126 \mathrm{MHz}$, $\left.\mathrm{CDCl}_{3}\right) \delta: 159.7,157.2,136.6,132.7,127.4,116.4,114.2$, 104.2, 55.3.

反, 反-1,4-二邻氟苯基-1,3-丁二烯(4f) ${ }^{[14]}$ : 白色固体, 收率 64\%, m.p. 140 142 ${ }^{\circ} \mathrm{C}$ (lit. ${ }^{[14]}$ no report); ${ }^{1} \mathrm{H}$ NMR $\left(500 \mathrm{MHz}, \mathrm{CDCl}_{3}\right) \delta: 7.33 \sim 7.28(\mathrm{~m}, 2 \mathrm{H}), 7.21(\mathrm{~d}, J=8.0$ $\mathrm{Hz}, 2 \mathrm{H}), 7.17 \sim 7.14(\mathrm{~m}, 2 \mathrm{H}), 6.97 \sim 6.91(\mathrm{~m}, 4 \mathrm{H}), 6.69 \sim$ $6.63(\mathrm{~m}, 2 \mathrm{H}) ;{ }^{13} \mathrm{C}$ NMR $\left(126 \mathrm{MHz}, \mathrm{CDCl}_{3}\right) \delta: 163.2(\mathrm{~d}$, $J=246.0 \mathrm{~Hz}), 139.5(\mathrm{~d}, J=8.0 \mathrm{~Hz}), 132.4(\mathrm{~d}, J=3.0 \mathrm{~Hz})$, $130.0(\mathrm{t}, J=9.0 \mathrm{~Hz}), 122.4(\mathrm{~d}, J=3.0 \mathrm{~Hz}), 114.6(\mathrm{~d}, J=$ $21.0 \mathrm{~Hz}), 112.7(\mathrm{~d}, J=22.0 \mathrm{~Hz})$.

反, 反-1,4-二对氟苯基-1,3-丁二烯 $(\mathbf{4 g})^{[18]}$ : 白色固 体, 收率 68\%, m.p. $165 \sim 168{ }^{\circ} \mathrm{C}$ (lit. ${ }^{[18]} 169{ }^{\circ} \mathrm{C}$ ); ${ }^{1} \mathrm{H}$ NMR $\left(500 \mathrm{MHz}, \mathrm{CDCl}_{3}\right) \delta: 7.42 \sim 7.39(\mathrm{~m}, 4 \mathrm{H}), 7.06 \sim$ $7.01(\mathrm{~m}, 4 \mathrm{H}), 6.88 \sim 6.82(\mathrm{~m}, 2 \mathrm{H}), 6.66 \sim 6.60(\mathrm{~m}, 2 \mathrm{H})$; ${ }^{13} \mathrm{C}$ NMR $\left(126 \mathrm{MHz}, \mathrm{CDCl}_{3}\right) \delta: 162.3(\mathrm{~d}, J=247.0 \mathrm{~Hz})$, 133.5 (d, $J=3.8 \mathrm{~Hz}), 131.6,128.8,127.4(\mathrm{~d}, J=7.6 \mathrm{~Hz})$, $115.6(\mathrm{~d}, J=21.4 \mathrm{~Hz}), 115.6$.

反, 反-1,4-二间氯苯基-1,3-丁二烯 $(\mathbf{4 h})^{[19]}$ : 黄色固 体, 收率 61\%, m.p. $115 \sim 117{ }^{\circ} \mathrm{C}$ (lit. ${ }^{[19]} 115{ }^{\circ} \mathrm{C}$ ); ${ }^{1} \mathrm{H}$ NMR $\left(500 \mathrm{MHz}, \mathrm{CDCl}_{3}\right) \delta: 7.45(\mathrm{~s}, 1 \mathrm{H}), 7.33 \sim 7.24(\mathrm{~m}$, $4 \mathrm{H}), 7.23 \sim 7.22(\mathrm{~m}, 2 \mathrm{H}), 6.98 \sim 6.94(\mathrm{~m}, 2 \mathrm{H}), 6.94 \sim 6.62$ $(\mathrm{m}, 2 \mathrm{H}), 6.86 \sim 6.84(\mathrm{~m}, 2 \mathrm{H}) ;{ }^{13} \mathrm{C}$ NMR $(126 \mathrm{MHz}$, $\left.\mathrm{CDCl}_{3}\right) \delta: 138.4,132.2,130.1,130.0,127.7,126.3,124.7$.

反, 反-1,4-二(2-噻吩基)-1,3-丁二烯(4i) ${ }^{[8]}$ : 白色固 体, 收率 35\%, m.p. 101 103 ${ }^{\circ} \mathrm{C}$ (lit. $\left.{ }^{[8]} 173{ }^{\circ} \mathrm{C}\right) ;{ }^{1} \mathrm{H}$ NMR $\left(500 \mathrm{MHz}, \mathrm{CDCl}_{3}\right) \delta: 7.18(\mathrm{~d}, J=6.0 \mathrm{~Hz}, 2 \mathrm{H}), 7.00 \sim 6.98$ $(\mathrm{m}, 4 \mathrm{H}), 6.79 \sim 6.67(\mathrm{~m}, 4 \mathrm{H}) ;{ }^{13} \mathrm{C}$ NMR $(126 \mathrm{MHz}$, $\left.\mathrm{CDCl}_{3}\right) \delta: 142.9,128.5,127.7,126.0,125.5,124.5$.

反, 反-1,4-二(2-吡啶基)-1,3-丁二烯(4j $)^{[19]}$ : 黄色固 体, 收率 30\%, m.p. $115 \sim 117{ }^{\circ} \mathrm{C}$ (lit. ${ }^{[19]}$ no report); ${ }^{1} \mathrm{H}$ NMR $\left(500 \mathrm{MHz}, \mathrm{CDCl}_{3}\right) \delta: 8.59(\mathrm{~d}, J=4.5 \mathrm{~Hz}, 2 \mathrm{H})$, $7.67 \sim 7.64(\mathrm{~m}, 2 \mathrm{H}), 7.50 \sim 7.47(\mathrm{~m}, 2 \mathrm{H}), 7.35(\mathrm{~d}, J=8.0$ $\mathrm{Hz}, 2 \mathrm{H}), 7.16 \sim 7.14(\mathrm{~m}, 2 \mathrm{H}), 6.86 \sim 6.84(\mathrm{~m}, 2 \mathrm{H}) ;{ }^{13} \mathrm{C}$ 
NMR $\left(126 \mathrm{MHz}, \mathrm{CDCl}_{3}\right) \delta: 155.3,149.7,136.6,134.2$, $132.5,122.4,122.3$.

反, 反-1,4-二(3-吡啶基)-1,3-丁二烯(4k) ${ }^{[19]}$ : 黄色固 体, 收率 $32 \%$, m.p. $124 \sim 126{ }^{\circ} \mathrm{C}$ (lit. ${ }^{[19]}$ no report); ${ }^{1} \mathrm{H}$ NMR (500 MHz, $\left.\mathrm{CDCl}_{3}\right) \delta: 8.67(\mathrm{~d}, J=2.0 \mathrm{~Hz}, 2 \mathrm{H})$, $8.50 \sim 8.48(\mathrm{~m}, 3 \mathrm{H}), 7.80 \sim 7.77(\mathrm{~m}, 2 \mathrm{H}), 7.31 \sim 7.28(\mathrm{~m}$, $2 \mathrm{H}), 7.04 \sim 7.01(\mathrm{~m}, 2 \mathrm{H}), 6.73 \sim 6.69(\mathrm{~m}, 2 \mathrm{H}) ;{ }^{13} \mathrm{C} \mathrm{NMR}$ $\left(126 \mathrm{MHz}, \mathrm{CDCl}_{3}\right) \delta: 148.7,148.5,132.8,130.6,130.2$, 123.7 .

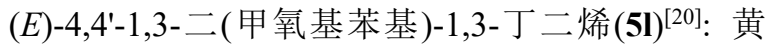
色油状液体, ${ }^{1} \mathrm{H}$ NMR $\left(400 \mathrm{MHz}, \mathrm{CDCl}_{3}\right) \delta: 7.27(\mathrm{~d}, J=$ $8.4 \mathrm{~Hz}, 2 \mathrm{H}), 7.14$ (d, $J=8.8 \mathrm{~Hz}, 1 \mathrm{H}), 6.77$ (q, $J=4.0 \mathrm{~Hz}$, $4 \mathrm{H}), 6.58$ (q, $J=6.8 \mathrm{~Hz}, 1 \mathrm{H}), 6.42$ (d, $J=12.8 \mathrm{~Hz}, 1 \mathrm{H})$, 5.53 (d, $J=15.6 \mathrm{~Hz}, 1 \mathrm{H}), 5.04$ (d, $J=15.2 \mathrm{~Hz}, 1 \mathrm{H}), 3.72$ $(\mathrm{d}, J=1.6 \mathrm{~Hz}, 6 \mathrm{H}) ;{ }^{13} \mathrm{C} \mathrm{NMR}\left(100 \mathrm{MHz}, \mathrm{CDCl}_{3}\right) \delta: 159.6$, $159.4,136.2,132.7,130.5,127.7,127.4,116.4,114.2$, $113.9,111.6,55.3$.

辅助材料(Supporting Information) 化合物 $4 \mathrm{a} \sim \mathbf{4 k}$ 和 $5 \mathrm{l}$ 的 ${ }^{1} \mathrm{H} \mathrm{NMR}$ 和 ${ }^{13} \mathrm{C} \mathrm{NMR}$ 图谱. 这些材料可以免费从本 刊网站(http://sioc-journal.cn/)上下载.

\section{References}

[1] (a) Ramamoorthy, G.; Acevedo, C. M.; Alvira, E.; Lipton, M. A. Tetrahedron: Asymmetry 2008, 19, 2546.

(b) Davis, R.; Kumar, N. S. S.; Abraham, S.; Suresh, C. H.; Rath, N. P.; Tamaoki, N.; Das, S. J. Phys. Chem. C 2008, 112, 2137.

(c) Xi, Z. F.; Zhang, W. X. Synlett 2008, 2557.

(d) Amans, D.; Bellosta, V. ; Cossy, J. Chem.-Eur. J. 2009, 15, 3457.

(e) Huang, J. M.; Dong, Y.; Wang, X. X.; Luo, H. C. Chem. Commun. 2010, 46, 1035.

(f) Zhou P.; Jiang, H. F.; Huang, L. B.; Li, X. W. Chem. Commun. 2011, 47, 1003.

(g) Wen, Y. M.; Huang, L. B.; Jiang, H. F. J. Org. Chem. 2012, 77, 5418.

(h) Li, W. B.; Zhang, J. L. Org. Lett. 2014, 16, 162

(i) Jiang, B.; Zhao, M.; Li, S. S.; Xu, Y. H.; Loh, T. P. Angew. Chem., Int. Ed. 2018, 57, 555.

[2] (a) Lemhadri, M.; Battace, A.; Berthiol, F.; Zair, T.; Doucet, H.; Santelli, M. Synthesis 2008, 1142.

(b) Blackwell, D. T.; Galloway, W. R. J. D.; Spring, D. R. Synlett 2011, 2140 .

(g) Yang, Q.; Chai, H.; Liu, T.; Yu, Z. Tetrahedron Lett. 2013, 54, 6485.

[3] (a) Phillips, A. M. F.; Pombeiro, A. J. L. ChemCatChem 2018, 10, 3354.

(b) Li, C.-J. Acc. Chem. Res. 2009, 42, 335.

(c) Varun, B. V.; Dhineshkumar, J.; Bettadapur, K. R.; Siddaraju, Y.; Alagiri, K.; Prabhu, K. R. Tetrahedron Lett. 2017, 58, 803.

(d) Wu, Y. N.; Wang, J.; Mao, F.; Kwong, F. Y. Chem.-Asian J.
2014, 9, 26.

(e) Ashenhurst, J. A. Chem. Soc. Rev. 2010, 39, 540.

(f) Lei, A. W.; Liu, W.; Liu, C.; Chen, M. Dalton Trans. 2010, 39, 10352

(g) Bras, J. L.; Muzart, J. Chem. Rev. 2011, 111, 1170.

(h) Liu, C.; Yuan, J. W.; Gao, M.; Tang, S.; Li, W.; Shi, R.; Lei, A. W. Chem. Rev. 2015, 115, 12138.

(i) Murakami, K.; Yamada, S.; Kaneda, T.; Itami K. Chem. Rev. 2017, 117, 9302 .

(j) Yeung, C. S.; Dong, V. M. Chem. Rev. 2011, 111, 1215.

(k) Wang, C. S.; Dixneuf, P. H.; Soulé, J. F. Chem. Rev. 2018, 118, 7532 .

(1) Li, X.; Ouyang, W., Nie, J.; Ji, S.; Chen, Q.; Huo, Y. ChemCatChem 2020, 12, 2358.

[4] (a) Wilklow-Marnell, M.; Li, B.; Zhou, T.; Krogh-Jespersen, K.; Brennessel, W. W.; Emge, T. J.; Goldman, A. S.; Jones, W. D. J. Am. Chem. Soc. 2017, 139, 8977.

(b) Sun, Q.; Cai, L.; Ding, Y.; Xie, L.; Zhang, C.; Tan, Q.; Xu, W. Angew. Chem., Intl. Ed. 2015, 54, 4549

(c) Wen, Y.; Xie, J.; Deng, C.; Wu, Y. Synlett 2015, 26, 1755.

[5] Denmark, S. E.; Tymonko, S. A. J. Am. Chem. Soc. 2005, 127, 8004.

[6] (a) Jiang, H. F.; Qiao, C. L.; Liu, W. B. Chem.-Eur. J. 2010, 16, 10968.

(b) Zhou P.; Jiang H. F.; Huang L. B. Chem. Commun. 2011, 47, 1003 .

[7] Wen, Y. M.; Huang, L. B.; Jiang, H. F. J. Org. Chem. 2012, 77, 5418.

[8] Itami, K.; Ushiogi, Y.; Nokami, T.; Ohashi, Y.; Yoshida, J. Org. Lett. 2004, 6, 3695.

[9] Miyaura, N.; Yamada, K.; Suginome, H.; Suzuki, A. J. Am. Chem. Soc. 1985, 107, 972.

[10] Dhital, R. N.; Sakurai, H. Asian J. Org. Chem. 2014, 3, 668.

[11] (a) Nagao, K.; Ohmiya, H.; Sawamura, M. Org. Lett. 2015, 17, 1304.

(b) Verma, A.; Snead, R. F.; Dai, Y.; Slebodnick, C.; Yang, Y.; Yu, H.; Yao, F.; Santos, W. L. Angew. Chem., Int. Ed. 2017, 56, 5111.

(c) Kojima, C.; Lee, K.-H.; Lin, Z.; Yamashita, M. J. Am. Chem. Soc. 2016, 138, 6662 .

(d) Morinaga, A.; Nagao, K.; Ohmiya, H.; Sawamura, M. Angew. Chem., Int. Ed. 2015, 54, 15859.

(e) Miralles, N.; Alam, R.; Szabó, K.J.; Fernández, E. Angew. Chem., Int. Ed. 2016, 55, 4303.

(f) Hong, S. B.; Zhang, W.; Liu, M. Y.; Yao, Z. J.; Deng, W. Tetrahedron Lett. 2016, 57, 1.

[12] Deng, C. M.; Ma, Y. F.; Wen, Y. M. ChemistrySelect 2018, 3, 1202.

[13] Zheng, C.; Wang, D.; Stahl, S. S. J. Am. Chem. Soc. 2012, 134, 16496.

[14] Thiel, N. O.; Kemper, S.; Teichert, J. F. Tetrahedron 2017, 73, 5023.

[15] Hintermann, L.; Schmitz, M.; Chen, Y. Adv. Synth. Catal. 2010, 352,2411

[16] Al-Jawaheri, Y.; Kimber, M. Org. Lett. 2016, 18, 3502.

[17] Zhuang, X.; Chen, J.-Y.; Yang, Z.; Jia, M.; Wu, C.; Liao, R.-Z.; Tung, C.-H.; Wang, W. Organometallics 2019, 38, 3752.

[18] Parrish, J. P.; Jung, Y. C.; Floyd, R. J.; Jung, K. W. Tetrahedron Lett. 2002, 43, 7899.

[19] Rodriguez, J. G.; Díaz-Oliva, C. Tetrahedron 2009, 65, 2512.

[20] Ting, C.-M.; Hsu, Y.-L.; Liu, R.-S. Chem. Commun. 2012, 48, 6577. 